\title{
Variante juvenil de la enfermedad de Sandhoff: presentación del primer caso descrito en Argentina
} Juvenile form of Sandhoff disease: first case reported in Argentina

\author{
Lic. Julia Mugnaini, ${ }^{a, b}$ Dra. Marcela Pereyra, ${ }^{c}$ Dra. Raquel Dodelson de Kremer, ${ }^{a}$ Dra. Beatriz Gamboni, ${ }^{d}$ \\ Dr. Carlos E. Argaraña, y yra. Ana M. Oller Ramírez ${ }^{a, b}$
}

\section{RESUMEN}

La enfermedad deSandhoffesuna patología neurodegenerativa, dealmacenamientolisosomal, causada por mutaciones en el gen HEXB. Existen tres formas clínicas: infantil, juvenil y adulta. Previamente, fue identificada una población endogámica en la provincia de Córdoba, Argentina, que presentaba una alta incidencia de la enfermedad; todos los casos correspondieron a la forma infantil. En este trabajo, se presenta por primera vez el caso de un paciente argentino con la variante juvenil de la enfermedad de Sandhoff. El paciente es un niño de 7 años que, a partir de los 2, presentó ataxia, trastorno del habla y retraso global en el desarrollo. El diagnóstico se confirmó con la detección de valores residuales de enzima hexosaminidasa y con la identificación de dos mutaciones ya descritas en estado de heterocigosis: c.796T >G (p.Y266D) y c.1615C > T (p.R539C). Palabras clave: gangliosidosis GM2, enfermedad de Sandhoff juvenil, gen HEXB.

\section{ABSTRACT}

Sandhoff disease is a neurodegenerative, lysosomal and autosomal recessive disease caused by mutations in the HEXB gene. Three forms are recognized: infantile, juvenile and

a. Centro de Estudio de las Metabolopatías Congénitas (CEMECO), Cátedra de Clínica Pediátrica, Facultad de Ciencias Médicas, Universidad Nacional de Córdoba(UNC), Córdoba, Argentina. Hospital de Niños de Córdoba.

b. Consejo Nacional de Investigaciones Científicas y Técnicas (CONICET), Argentina.

c. Servicio de Crecimiento y Desarrollo, Hospital Pediátrico Dr. H. J. Notti, Mendoza, Argentina.

d. Servicio de Neurología, Hospital Pediátrico Dr. H. J. Notti, Mendoza, Argentina.

e. Centro deInvestigaciones en Química Biológica de Córdoba (CIQUIBIC-CONICET), Facultad de Ciencias Químicas, Universidad Nacional de Córdoba (UNC), Córdoba, Argentina.

Correspondencia:

Lic. Julia Mugnaini: juliamugnaini@gmail.com

\begin{abstract}
Financiamiento: Este trabajo fue realizado gracias al apoyo económico de la Secretaría de Ciencia y Tecnología de la Universidad Nacional de Córdoba (SECyT, Resolución N. $\left.{ }^{\circ} 203 / 14\right)$ y de la Agencia Nacional de Promoción Científica y Tecnológica (FONCyT, PICT 2010-1116 N. ${ }^{\circ} 2437$ /OC-AR, Resolución N. ${ }^{\circ}$ 098/11).

Conflicto de intereses: Ninguno que declarar.
\end{abstract}

Recibido: 22-12-2016

Aceptado: 12-6-2017 adult. Previously, an endogamous population in Córdoba, Argentina, was identified with a high incidence of Sandhoff disease, all reported cases were of the infantile type. In this work, we describe a child with the juvenile form of Sandhoff disease, the first case reported in Argentina. The patient is a 7-year-old boy presenting with ataxia, speech disturbances and global developmental delay, symptoms starting at the age of 2 years. Diagnosis was based on the hexosaminidase deficiency. Sequencing of genomic DNA revealed compound heterozygosity for two HEXB gene mutations: c.796T $>$ G (p.Y266D) and c.1615C>T (p.R539C), both already reported. Key words: GM2 gangliosidoses, juvenile Sandhoff disease, HEXB gene.

http: / / dx.doi.org/10.5546/ aap.2017.e298

Cómo citar: Mugnaini J, Pereyra M, Dodelson de Kremer R, et al. Variante juvenil de la enfermedad de Sandhoff: presentación del primer caso descrito en Argentina. Arch Argent Pediatr 2017;115(5):e298-e301.

\section{INTRODUCCIÓN}

La enfermedad de Sandhoff (ES) o gangliosidosis GM2 tipo II (MIM 268800) es una enfermedad de almacenamiento lisosomal, caracterizada, principalmente, por el acúmulo progresivo de gangliósido GM2 a nivel neuronal. Tiene un patrón de herencia autosómico recesivo. ${ }^{1,2}$

En esta patología, existe un defecto en el complejo lisosomal de las enzimas hexosaminidasas (Hex); las principales son Hex A $(\alpha \beta)$ y Hex B $(\beta \beta)$. En la ES, se encuentra mutado el gen $H E X B$ que codifica la subunidad $\beta$, por lo tanto, Hex A y Hex B resultan afectadas. ${ }^{1,2}$

La ES se caracteriza por presentar un cuadro neurodegenerativo progresivo. Existen 3 formas clínicas principales. Variante infantil: con aparición temprana de la enfermedad, entre 3 y 6 meses de edad, deterioro progresivo motor y mental, ceguera precoz y evolución fatal a los 20 3 años de vida. ${ }^{1,3}$ Variante juvenil: presenta gran heterogeneidad clínica; los primeros síntomas aparecen entre los 2 y los 10 años, y los más comunes son dificultades en la marcha, trastorno del habla, falta de coordinación y retraso global en el desarrollo. La muerte ocurre entre los $10 \mathrm{y}$ 
los 15 años de vida. ${ }^{14}$ Variante adulta o crónica: existe una extremada variabilidad clínica; la aparición de síntomas es tardía y puede ocurrir entre la infancia y la adultez. Puede presentarse con distonía, degeneración espinocerebelosa, enfermedad de la motoneurona, alteraciones psiquiátricas, entre otras. La supervivencia en estos pacientes puede alcanzar la sexta o séptima década de vida. ${ }^{1,5}$

La ES es una patología poco frecuente en la población en general, con una incidencia estimada de 1 enfermo cada 422000 individuos. ${ }^{4}$ Sin embargo, esta enfermedad fue reconocida con alta incidencia en diferentes grupos étnicos, y se destaca la casuística de la comunidad criolla del Valle de Traslasierra de Córdoba y los llanos de La Rioja, Argentina. ${ }^{3,6,7}$ En esta población endogámica, la frecuencia de portadores fue estimada en 1 cada 16-29 individuos y la incidencia de enfermos, de $131 \times 10^{-5}$ (100000 nacimientos), equivalente a 1 enfermo cada 763 recién nacidos (RN). ${ }^{7}$ Todos los casos hasta la fecha pertenecieron a la forma infantil de la enfermedad. En este trabajo, se presenta por primera vez la descripción clínica, bioquímica y molecular de un paciente argentino con variante juvenil de la ES.

\section{PRESENTACIÓN DEL CASO CLÍNICO}

Paciente de 7 años de edad, sexo masculino, nacido en Mendoza, que consultó a los 2 años al Servicio de Neurología del Hospital Pediátrico Dr. Notti por presentar pérdida de pautas madurativas, desconexión con el medio, ataxia y trastorno del lenguaje.

Antecedentes: primer y único hijo de una pareja sana, no consanguínea, sin antecedentes de importancia. La familia era originaria de Mendoza, no refería parentesco con la población de Traslasierra de Córdoba.

Embarazo controlado. A las 40 semanas de gestación, cesárea electiva por distocia materna (pelvis estrecha). Peso del RN: 3,650 kg; talla: $50 \mathrm{~cm}$; perímetro cefálico (PC): $34 \mathrm{~cm}$; Apgar 8-9.

Desarrollo: retraso en la adquisición de pautas madurativas y, a los 2 años, pérdida de las adquiridas. Dejó de caminar, con retraso muy importante en el lenguaje, hipotonía, babeo, facies amímica y trastorno del espectro autista. Signos piramidales.

Al momento del examen físico, a la edad de 5 años, se destacó baja talla y macrocefalia relativa. Peso: $17,900 \mathrm{~kg}$; talla: $100,9 \mathrm{~cm}$; PC: 51,6 cm; e índice de masa corporal (IMC): $17,58 \mathrm{~kg} / \mathrm{m}^{2}$.
Exámenes complementarios: estudio de fondo de ojo normal, sin presencia de mancha rojocereza. Potenciales evocados auditivos normales (a los 5 y 7 años). Las imágenes de la resonancia magnética nuclear (RMN) de cerebro a los 4 años revelaron hiperintensidad, en T2 y FLAIR, de sustancia blanca subcortical y periventricular e imágenes puntiformes a nivel subcortical de la región frontal izquierda y el tronco cerebral. También se destacó adelgazamiento del cuerpo calloso. Sin compromiso del cerebelo.

Se realizaron estudios de laboratorio (función hepática, renal, tiroidea, perfil lipídico, metabolismo fosfocálcico, glucemia), todo dentro de los límites de referencia, a excepción de los valores de hemoglobina por debajo de lo normal.

Ante la sospecha de una patología de almacenamiento lisosomal, se solicitaron estudios bioquímicos específicos y se llegó al diagnóstico de ES. La determinación de la enzima Hex en el plasma por el método fluorogénico con inactivación térmica confirmó la deficiencia enzimática. El valor detectado de la Hex total (Hex A + Hex B) fue de 16,52 nmol $/ \mathrm{ml} / \mathrm{h}(2,65 \%$, valor normal $-\mathrm{VN}-=400-1150 \mathrm{nmol} / \mathrm{ml} / \mathrm{h}$ ) y el de Hex B fue $0,36 \mathrm{nmol} / \mathrm{ml} / \mathrm{h}(0,13 \%, \mathrm{VN}=140$ $600 \mathrm{nmol} / \mathrm{ml} / \mathrm{h}$ ).

Se realizó la secuenciación del gen $H E X B$, que incluyó el análisis exhaustivo de sus 14 exones. Se identificaron 2 cambios de tipo missense: c.796T $>\mathrm{G}$ (p.Y266D) en el exón 7 y c.1615C>T (p.R539C) en el exón 14 (Figura 1). Estas mutaciones han sido previamente reportadas en pacientes con $\mathrm{ES}$, tanto en la forma infantil como en la juvenil. El análisis genético también se realizó a nivel familiar y confirmó el estado de portador en la madre (heterocigota p.R539C) y el padre (heterocigota p.Y266D).

A los 5 años y 5 meses, el paciente presentó autoagresiones y fue medicado con risperidona.

Inició un tratamiento a los 6 años y 5 meses con miglustat (Zavesca ${ }^{\circledR}$ y dieta con disminución de disacáridos. Se observó una mejora en la hipotonía, desplazamiento con gateo y mayor conexión con el observador.

Examen físico actual, a los 7 años, con peso de $17,800 \mathrm{~kg}$, talla de $104,8 \mathrm{~cm}$, PC de 51,5 cm e IMC de $16,2 \mathrm{~kg} / \mathrm{m}^{2}$. Mejor tono muscular; se desplaza gateando. Lenguaje: gritos.

\section{DISCUSIÓN}

La ES en su forma juvenil es una patología poco frecuente a nivel mundial. En los últimos años, se han publicado 39 casos de 
pacientes con diversos orígenes étnicos, entre ellos, paquistaníes, japoneses, brasileros, italianos, españoles, franceses, canadienses y estadounidenses. ${ }^{4,8-15}$ En Argentina, si bien existe la población endogámica con alta incidencia de ES infantil, hasta ahora, ningún paciente había tenido diagnóstico de ES juvenil. 3,6,7

Maegawa y col., en 2006, publicaron una revisión de la historia natural de las gangliosidosis GM2 variante juvenil (enfermedades de TaySachs y Sandhoff) y analizaron 21 casos nuevos y 134 pacientes previamente descritos en la bibliografía. La edad de inicio de los síntomas para casos nuevos y para pacientes previamente publicados se ubicaba en el rango de 2 a 15 años y entre 1 y 17 años, respectivamente. Los síntomas comunes descritos fueron dificultad en la marcha, trastornos del habla y lenguaje, falta de coordinación, discapacidad intelectual y signos piramidales. Otros hallazgos clínicos identificados con menor frecuencia fueron debilidad muscular, incontinencia urinaria y/o fecal, deformidades del pie, alteraciones psiquiátricas o de comportamiento, disfagia, signos extrapiramidales, pobre ganancia de peso, convulsiones y disminución visual. En el presente

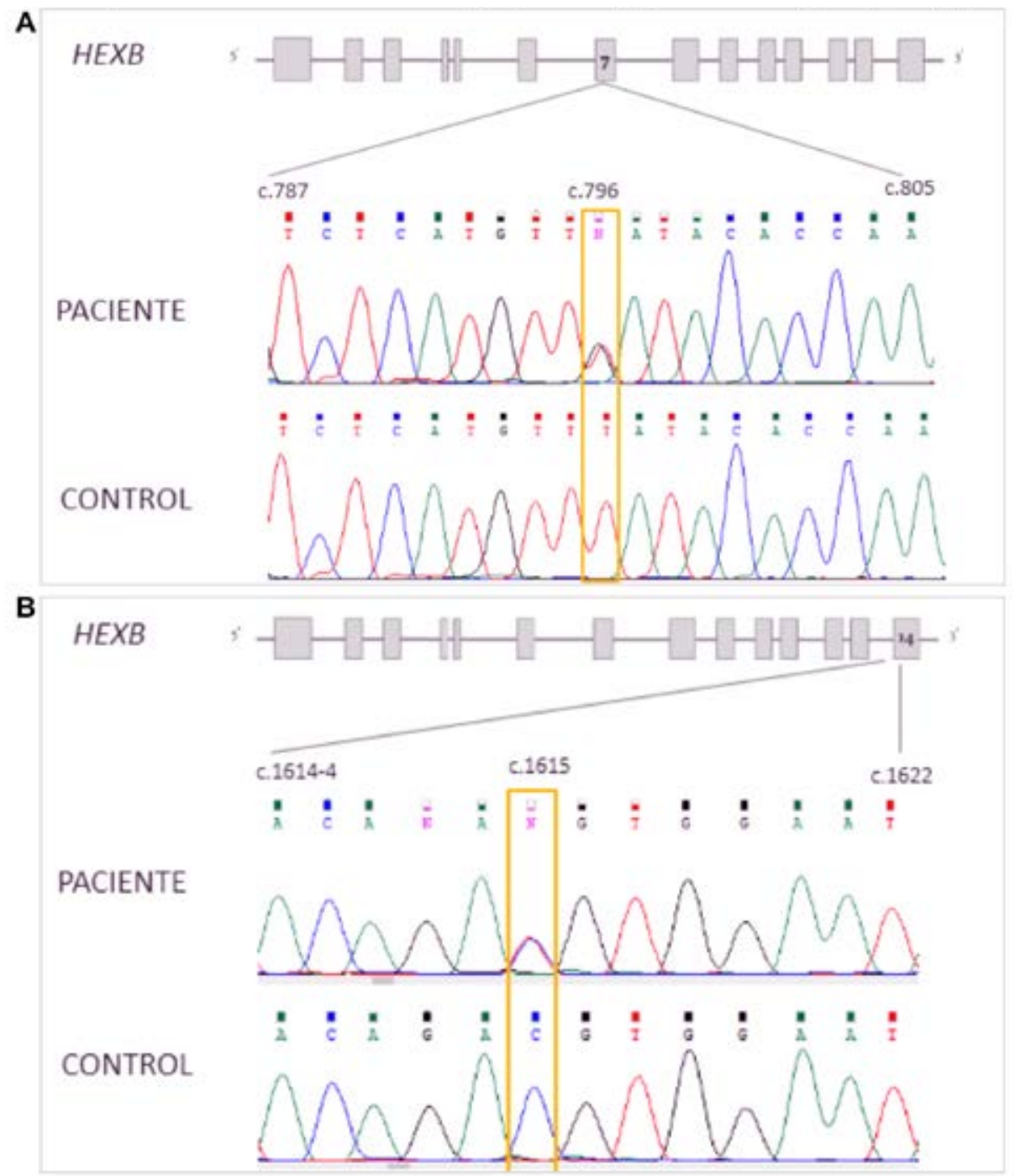

Electroferogramas correspondientes a fragmentos del gen HEXB en el paciente y en un control.

A. Parte del exón 7. En la secuencia del paciente, se observa el doble pico en posición c.796, cambio T>G en heterocigosis.

B. Porción del intrón 13 y exón 14. Doble pico en posición c.1615, cambio C>T en heterocigosis. 
caso clínico, tanto la edad de inicio de síntomas como los hallazgos clínicos más comunes se correspondieron con lo mencionado en el trabajo de Maegawa y col. Entre los signos menos frecuentes, el paciente presentó alteraciones psiquiátricas y trastorno del espectro autista. ${ }^{4}$

En los exámenes complementarios, Maegawa y col. destacaron, como hallazgos principales, la atrofia tanto cerebelar como cerebral y cambios en la sustancia blanca subcortical observados en las imágenes de RMN. ${ }^{4}$ Si bien, en el paciente no se observaron alteraciones cerebelares, sí fue evidente la atrofia cerebral y la alteración de la sustancia blanca. Además, la mancha rojo-cereza en la mácula de la retina, signo presente en casi todos los enfermos con gangliosidosis GM2 infantil, no fue identificada en el paciente, lo que coincidió con la mayoría de los casos clínicos juveniles reportados..$^{1,4,8}$

En cuanto a los resultados a nivel enzimático, el porcentaje de actividad Hex total detectado en el paciente, $2,65 \%$, fue comparable con los resultados publicados para otros pacientes con ES juvenil, cuyos valores oscilaron entre $0,5 \%$ y $8,3 \%$.,10,12

Las mutaciones identificadas en este caso clínico, p.Y266D y p.R539C, han sido previamente descritas en la bibliografía. La variante p.Y266D fue registrada en 5 enfermos, de los cuales 3 se correspondieron con la forma infantil de la enfermedad y 2 con la variante juvenil. ${ }^{4,9,10}$ Además, el cambio p.R539C solo fue identificado en un paciente caucásico para el que no hay datos acerca de la edad de diagnóstico, forma clínica ni edad de fallecimiento. ${ }^{10}$ No existen registros previos de pacientes argentinos que hayan presentado estas mutaciones.

Dado que, en este trabajo, se presenta un único caso clínico, la información disponible no es suficiente para establecer qué tipo de relación existe entre el genotipo identificado y el fenotipo resultante, ni acerca de cómo estas mutaciones pueden impactar sobre la actividad enzimática de la Hex.

La sumatoria de las características clínicas, bioquímicas y moleculares nos permitieron concluir que el paciente presentó la variante juvenil de la ES y es el primer caso publicado en Argentina.

\section{Agradecimientos}

A la Bioq. Ana María Guercio por colaborar con la toma de muestras y la derivación a CEMECO.

\section{REFERENCIAS}

1. Gravel RA, Kaback MM, Proia RL, et al. The $G_{M 2}$ Gangliosidoses. In Scriver CR (edit). The Metabolic and Molecular Basis of Inherited Disease. New York: McGrawHill, 2001:3827-76.

2. Mahuran DJ. Biochemical consequences of mutations causing the GM2 gangliosidoses. Biochim Biophys Acta 1999; 1455(2-3):105-138.

3. Dodelson de Kremer R, Molina de Levstein I. Enfermedad de Sandhoff o Gangliosidosis GM2 tipo 2. Alta frecuencia del gen en una población criolla. Medicina (B Aires) 1980;40(1):55-73.

4. Maegawa GHB, Stockley T, Tropak M, et al. The natural history of juvenile or subacute GM2 gangliosidosis: 21 new cases and literature review of 134 previously reported. Pediatrics 2006;118(5):e1550-62.

5. Schnorf H, Gitzelmann R, Bosshard NU, et al. Early and severe sensory loss in three adult siblings with hexosaminidase A and B deficiency (Sandhoff disease). J Neurol Neurosurg Psychiatry 1995;59(5):520-3.

6. Kremer R, Boldini C, Capra A, et al. Sandhoff disease: 36 cases from córdoba, Argentina. J lnherit Metab Dis 1985;8(1):46.

7. Doleson de Kremer R, Depetris de Boldini C, Paschini de Capra A, et al. Estimación de la frecuencia heterozigótica de la enfermedad de Sandhoff en una población argentina dealto riesgo. Asignación predictiva del genotipo mediante análisis estadístico. Medicina (B Aires) 1987;47(5):455-63.

8. Hendriksz CJ, Corry PC, Wraith JE, et al. Juvenile Sandhoff disease--nine new cases and a review of the literature. J Inherit Metab Dis 2004;27(2):241-9.

9. Gaignard P, Fagart J, Niemir N, et al. Characterization of seven novel mutations on the HEXB gene in French Sandhoff patients. Gene 2013;512(2):521-6.

10. GortL,DeOlanoN,Macías-VidalJ, etal.GM2 gangliosidoses in Spain: Analysis of the HEXA and HEXB genes in 34 TaySachs and 14 Sandhoff patients. Gene 2012;506(1):25-30.

11. Mitsuo K, Nakano T, Kobayashi T, et al. Juvenile Sandhoff disease: a Japanese patient carrying a mutation identical to that found earlier in a Canadian patient. J Neurol Sci 1990;98(2-3):277-86.

12. ZampieriS, CattarossiS, Oller Ramirez AM, et al. Sequence and copy number analyses of HEXB gene in patients affected by Sandhoff disease: Functional characterization of 9 novel sequence variants. PLoS One 2012;7(7):e41516.

13. Pierson TM, Torres PA, Zeng B-J, et al. Juvenile-onset motor neuron disease caused by novel mutations in $\beta$-hexosaminidase. Mol Genet Metab 2012;108(1):65-9.

14. Nakano T, Suzuki K. Genetic cause of a juvenile form of Sandhoff disease. Abnormal splicing of betahexosaminidase beta chain gene transcript due to a point mutation within intron 12. J Biol Chem 1989;264(9):5155-8.

15. Kytzia HJ,Hinrichs U, SandhoffK. Diagnosis of infantile and juvenile forms of GM2 gangliosidosis variant 0 . Residual activities toward natural and different synthetic substrates. Hum Genet 1984;67(4):414-8. 